\title{
Atención ginecológica de lesbianas y bisexuales: notas sobre el estado de situación en Argentina
}

\author{
Josefina Leonor Brown ${ }^{(a)}$ \\ Mario Pecheny ${ }^{(b)}$ \\ María Cecilia Tamburrino(c) \\ Leandro Luciani Conde ${ }^{\text {(d) }}$ \\ Gabriela Viviana Perrotta(e) \\ Alejandro Capriati ${ }^{(f)}$ \\ Ana María Andia(g) \\ Silvia Mario(h) \\ Inés Ibarlucia ${ }^{(i)}$
}

Brown JL, Pecheny M, Tamburrino MC, Conde LL, Perrotta GV, Capriati A, et al. Gynecological care among lesbians and bisexual women: notes on the situation in Argentina. Interface (Botucatu). 2014; 18(51):673-84.

This paper presents results from a qualitative study on emerging issues within sexual and reproductive health, in which the aim was to describe the experiences of non-heterosexual women in relation to sexual and reproductive healthcare services. The objective was to identify and analyze perceptions of obstacles and facilitators of access to healthcare services. Eighteen semi-directed interviews were conducted with non-heterosexual women. The main result was that the specific obstacle to access to sexual and reproductive healthcare among these non-heterosexual women derived from a "logic of invisibility" among this population as gynecological patients, which resulted in anticipated discrimination (sensed discrimination) within a homo/lesbophobic context; absence of theoretical production and practical interventions regarding health and sex between women; and invisibility of lesbians and bisexuals as a group.

Keywords: Sexuality. Reproduction. Reproductive Health Services. Bisexuality. Female homosexuality.
El artículo resume algunos resultados de la investigación sobre "Salud sexual y reproductiva: problemas emergentes", cuyo objetivo fue describir las experiencias de mujeres no heterosexuales, en sus vínculos con los servicios de salud sexual y reproductiva, buscando identificar y analizar cuáles son los factores percibidos como obstaculizadores o facilitadores para su acceso. Partiendo de una estrategia cualitativa se realizaron 18 entrevistas semiestructuradas a mujeres no heterosexuales. Como resultado encontramos que el mayor obstáculo para el acceso a servicios de salud sexual y reproductiva de mujeres no heterosexuales estriba en las "lógicas de invisibilización" que se traduce en la discriminación sentida en el marco de un contexto homo/lesbofóbico, la falta de producción teórica y la ausencia de preocupación práctica sobre relaciones sexuales entre mujeres y la invisibilidad de las lesbianas y bisexuales como colectivo.

Palabras clave: Sexualidad. Reproducción. Servicio de salud sexual y reproductiva. Bisexualidad. Homosexualidad femenina.

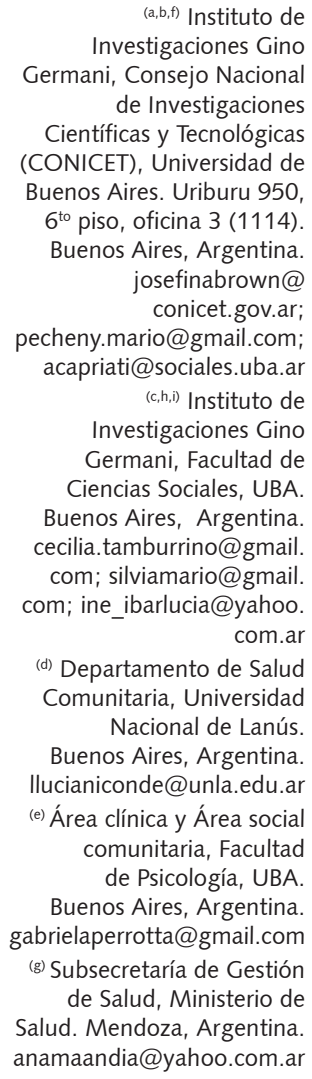

$(a, b, f)$ Instituto de Investigaciones Gino Germani, Consejo Nacional de Investigaciones Científicas y Tecnológicas (CONICET), Universidad de Buenos Aires. Uriburu 950, $6{ }^{\text {to }}$ piso, oficina 3 (1114). Buenos Aires, Argentina. josefinabrown@ conicet.gov.ar; pecheny.mario@gmail.com; acapriati@sociales.uba.ar $(\mathrm{c}, \mathrm{h}, \mathrm{i})$ Instituto de Investigaciones Gino Germani, Facultad de Ciencias Sociales, UBA. Buenos Aires, Argentina. cecilia.tamburrino@gmail. com; silviamario@gmail. com; ine_ibarlucia@yahoo. com.ar

(d) Departamento de Salud Comunitaria, Universidad Nacional de Lanús. Buenos Aires, Argentina. llucianiconde@unla.edu.ar (e) Área clínica y Área social comunitaria, Facultad de Psicología, UBA. Buenos Aires, Argentina. gabrielaperrotta@gmail.com (g) Subsecretaría de Gestión de Salud, Ministerio de Salud. Mendoza, Argentina. anamaandia@yahoo.com.ar 


\section{Introducción}

En Argentina, los programas de salud sexual y procreación responsable enfatizan, en sus políticas, el aspecto reproductivo de los derechos sexuales, reproductivos y no reproductivos ${ }^{1}$. La presunción de heterosexualidad reproductiva hace de las mujeres heterosexuales en edad reproductiva las principales destinatarias de esas políticas que marginaliza a varones heterosexuales o no, por un lado y a personas no heterosexuales (mujeres y trans), por otro. En ese contexto, existe escaso conocimiento de cuál es el grado de atención o no de lesbianas y bisexuales en el sistema de salud y, menos aún, en lo referido a los servicios de ginecología y obstetricia. A cubrir ese vacío se abocó la investigación "Salud sexual y reproductiva: problemas emergentes", parte de cuyos resultados presentamos aquí ${ }^{2}$. El objetivo fue describir las experiencias de mujeres no heterosexuales -lesbianas y bisexuales-, en sus vínculos con los servicios de salud sexual y (no) reproductiva, buscando identificar y analizar cuáles son los factores percibidos como obstaculizadores o facilitadores para su acceso.

\section{Hipótesis de trabajo}

Además del heterosexismo naturalizado y de la desigualdad intrínseca a la relación médico/a - paciente es posible localizar como uno de los mayores obstáculos en el acceso a servicios de ginecología y obstetricia la discriminación sentida en el marco de un contexto homo/lesbofóbico, la falta de producción teórica y la ausencia de preocupación práctica sobre relaciones sexuales entre mujeres y la invisibilidad de las lesbianas y bisexuales como colectivo. Resumimos esas variables bajo el término políticas o lógicas de invisibilización ${ }^{3-5}$ ): aquellas que niegan u ocultan a las mujeres no heterosexuales y determinadas prácticas sexuales que suelen corresponderles mayoritariamente aunque no exclusivamente (considerando que no hay una adecuación bis a bis entre prácticas sexuales e identidad sexual). La invisibilidad del colectivo lésbico está siendo creciente problematizada no sólo en el ámbito social más amplio sino incluso dentro mismo del colectivo de la diversidad sexual. Tal cuestionamiento puede apreciarse en la revisión que realizara ${ }^{6}$ en la que, a pesar de la declaración sobre una realización del estado el arte de la salud de bisexuales, lesbianas y homosexuales, prácticamente no aparece nada específico de la población lesbiana y menos aún de su salud ginecológica. Por ello es que diversos estudios han comenzado a realizarse en la última década en Latinoamérica en general y Argentina en particular para visibilizar su existencia, temas y problemas como lo muestran recientes investigaciones ${ }^{5-9}$.

Estas políticas de la invisibilidad homoérotica femenina juegan un papel central en el marco concreto de la consulta ginecológica derivando en una inadecuada atención sobre necesidades de las mujeres (cualquiera sea su orientación sexual) que excedan la sexualidad coitocéntrica heterosexista reproductiva en el marco de parejas monogámicas tal como fuera denunciado en diversas oportunidades por activistas. La denuncia sobre la deficiente atención ginecológica de lesbianas y bisexuales fue compilada en una edición que recoge algunos datos no sistemáticos de las experiencias de lesbianas con los servicios de salud en Argentina y a partir de ello propone que "Cambiemos las preguntas" 10 .

\section{Algunas nociones clave: precisiones}

Entendemos por accesibilidad a las condiciones materiales y simbólicas que facilitan u obstaculizan el acceso a prácticas saludables y servicios de salud, de sujetos individuales y colectivos, reconociendo su diversidad en términos cognitivos, valorativos y de recursos derivados de diferencias socioeconómicas, sexuales, etarias, educativas y geográficas. Las condiciones materiales y simbólicas se traducen en factores subjetivos y objetivos de accesibilidad, cuya operacionalización requiere focalizarse en dimensiones propias del sistema de salud $u$ otras que lo exceden. En particular, por tratarse de una investigación en salud sexual y reproductiva, las dimensiones interrogadas incluyen aspectos relativos al vínculo entre salud, sexualidad, género y reproducción. 
Nos referiremos a lesbianas y bisexuales respetando la autonominación de las entrevistadas. Se trata de mujeres no heterosexuales que construyen sus prácticas sexuales, eróticas, amorosas y/o afectivas en relación con otras mujeres - sea o no de modo exclusivo - e incluya o no una identidad como lesbiana, mujer bisexual u otra identidad. A los efectos de este proyecto, se trata de mujeres "biológicas", es decir que no incluye a quienes se identifican como mujeres habiendo transitado un proceso de identidad "trans" (travesti, transgénero).

\section{Metodología}

Las poblaciones mujeres - lesbianas y bisexuales- fueron contactadas a partir de una definición muestral según criterios de muestreo teórico y factibilidad, en tres áreas urbanas del país (Ciudad de Buenos Aires, Gran Buenos Aires y Mendoza).

Dada la especificidad de la temática, la escasez de investigaciones previas y el tiempo y presupuesto establecidos para su desarrollo, se trató de un diseño de tipo exploratorio-descriptivo. Se priorizó el estudio de las variables en relación con la especificidad de los casos. Es decir, no se profundizó el estudio de variables más generales sobre accesibilidad al sistema de salud ni sobre accesibilidad a la salud sexual y reproductiva. Se tomaron principalmente en cuenta pues los aspectos específicos de la interrogación que guío la investigación. Se siguió un criterio de muestreo teórico determinado a su vez por la factibilidad de contacto de entrevistadas.

La muestra está compuesta de 18 mujeres. Doce de ellas viven en el Área Metropolitana de Buenos Aires (AMBA) y las otras 6 residen en la Ciudad de Mendoza. De las que residen en AMBA, seis se nombran a sí mismas como lesbianas, una como gay y las otras cinco como bisexuales. Mientras que en Mendoza el total se autodenominó lesbiana.

La edad de las entrevistadas va de los 21 a los 33 años, con la excepción de una de ellas que tiene 44 años; es decir, se trata de jóvenes adultas, todas ellas en edad reproductiva.

En cuanto al nivel educativo, la mayor parte de ellas tiene como nivel educativo máximo el universitario incompleto (16 sobre 18). Las dos restantes, una tiene nivel terciario completo y la otra, un posgrado finalizado.

La mayor parte de las entrevistadas cuenta con obra social o prepaga propia, de la pareja o de los padres. Tres casos usan el sistema público de atención sanitaria.

En relación con el nivel de empleo, cuatro de las entrevistadas se encuentran desempleadas; una es estudiante exclusiva, otra depende económicamente de su pareja y otra trabaja en condiciones de precariedad. Las once restantes trabajan en empleos estatales, empleos privados en relación de dependencia o de manera independiente.

En cuanto a la relación de pareja, doce de las entrevistadas están casadas (post-ley de matrimonio igualitario ${ }^{(j)}$, conviviendo, de novias o en parejas estables con mujeres. Dos están en parejas estables con varones; dos "están saliendo" con un varón de manera informal, y dos no tienen pareja.

La mayor parte de las entrevistadas se identifican como lesbianas y la mayoría se encuentra en pareja: esas dos características coinciden con los datos de la encuesta realizada en la marcha del Orgullo en Buenos Aires en 2005. De acuerdo con los resultados de dicha encuesta, las lesbianas manifiestan encontrarse en pareja en mayor proporción que las otras categorías sexuales no heterosexuales relevadas (gays y travestis) ${ }^{11}$. 
Se trata de un estudio cualitativo, por lo cual no se apunta a relaciones causales y/o estadísticamente significativas, sino significativas en términos de los sentidos socialmente construidos.

Para responder a los objetivos y preguntas de la investigación, el instrumento de recolección de datos fue la entrevista semi-estructurada, basada en una guía de preguntas y temas, sobre la cual se elaboró un manual de códigos (siguiendo los ejes temáticos de la guía y otros emergentes del análisis). Para procesar el corpus de entrevistas se utilizó una grilla de análisis o sistematización.

Con el fin de acceder a la red de significaciones y discursos de las mujeres protagonistas que permitiera comprender los factores que obstaculizan o facilitan el acceso a los servicios de salud sexual y reproductiva para mujeres lesbianas y bisexuales, se hicieron entrevistas semi-estructuradas a mujeres lesbianas y bisexuales en edad reproductiva, así como a profesionales de la salud involucrados con la temática objeto de la investigación.

El análisis de los datos correspondientes al corpus constituido por las entrevistas realizadas en el marco de este estudio, se realizó según enfoques procedimentales ${ }^{12,13}$, que incluyeron: a) tareas de reducción de datos; b) disposición y transformación de los datos; y c) extracción/verificación de resultados. El procesamiento de estos datos cualitativos se llevó a cabo de manera manual.

a) Reducción de datos.

Las actividades a través de las cuales se llevó a cabo la reducción de los datos fueron:

a1. Separación en unidades: los segmentos o unidades de interés dentro del conjunto global de datos se diferenciaron según criterios gramaticales. Se trabajó con párrafos como "unidades de contexto", en tanto que las "unidades de registro" quedaron constituidas por aquellos contenidos específicos que se ubican en las categorías empleadas.

a2. Identificación y clasificación de unidades: las tareas de categorización y codificación se realizaron según procedimientos mixtos inductivo-deductivos, puesto que algunas categorías fueron definidas a priori en función del "proyecto anticipatorio", y otras emergieron a partir del trabajo de interpretación.

a3. Síntesis y agrupamiento: las distintas unidades fueron organizadas en función de los temas conceptuales que las representan y en relación al sistema de categorías empleado ${ }^{14}$. El sistema de categorías se construyó teniendo en cuenta los siguientes criterios: exhaustividad, exclusión mutua (para las unidades de contexto), principio clasificatorio único, significatividad y replicabilidad.

b) Disposición y transformación de los datos:

Se confeccionaron matrices de datos mediante los cuales se dispusieron y organizaron los datos con la finalidad de favorecer el trabajo interpretativo.

c) Extracción, verificación de resultados:

A partir de la matriz de análisis mencionada en el punto anterior, se fueron elaborando los resultados y conclusiones.

Los resultados presentados expresan las opiniones y valoraciones de las mujeres y de los profesionales y referentes de Programa entrevistadas/os, y no pueden ser generalizadas sin mediaciones al conjunto de ninguna población.

En la realización de la investigación se respetaron las normas éticas establecidas: todos los nombres de las entrevistadas y entrevistados, así como cualquier otro dato que pudiera identificarlos, fueron modificados para preservar la confidencialidad de datos sensibles. Asimismo antes de realizar las entrevistas se informó sobre el objeto de estudio, la confidencialidad y el uso posterior de los datos y se elaboró un consentimiento informado.

\section{Resultados}

\section{La atención ginecológica en mujeres}

En consonancia con los datos arrojados por una investigación previa, en las mujeres que entrevistamos una serie de variables (el ser jóvenes adultas, sanas, educadas, con buena cobertura de salud, residentes en grandes centros urbanos, etcétera) haría de ellas, las candidatas a una excelente 
accesibilidad en relación con los servicios de salud sexual y reproductiva ${ }^{15}$. Si para el caso de las heterosexuales los puntos límites que permiten ver más claramente obstaculizadores y facilitadores en el acceso a la atención ginecológica tienen que ver con la maternidad o el aborto, aquí el contrapunto está dado por la orientación sexual de las entrevistadas. Corroborando aquellos hallazgos así como otros algunas investigaciones latinoamericanas ${ }^{16}$ también aquí encontramos la consulta ginecológica es pensada en términos de obligaciones femeninas básicas, como si viniera de suyo con el cuerpo que se porta que a una edad, la de "hacerse adulta" (vía menarca o relación sexual) hubiera que "controlarse". Un control vinculado con la creciente medicalización y vigilancia de los cuerpos femeninos ligado a su vez a la idea de salud sexual y reproductiva, al menos tal y como se traduce en la legislación y las políticas públicas: es decir, la salud sexual es pensada en términos de planificación familiar. Se enfatiza el aspecto reproductivo y los cuidados necesarios del cuerpo femenino y su aparato génito mamario; $y$, en esa línea la ginecología apunta a proveer anticoncepción para que la maternidad llegue en el momento que el saber experto considera más apropiado así como para permitir los espaciamientos adecuados de los embarazos ${ }^{1,17}$.

\section{La atención sexual y reproductiva de lesbianas y bisexuales}

Entre las entrevistadas distinguimos dos grupos: mujeres que asisten regularmente al ginecólogo/a y mujeres que fueron muy pocas veces o nunca. Este último grupo está compuesto de 6 mujeres lo que equivale al $33 \%$ de la muestra y se corresponde con el porcentaje de no asistencia a los servicios de ginecología detectado por estudios previos. De acuerdo con una encuesta realizada en el año 2005 a asistentes a la Marcha del Orgullo Gay en la ciudad de Buenos Aires el 70,3\% de las lesbianas y el $71,4 \%$ de las mujeres bisexuales dicen asistir por lo menos una vez por año al ginecólogo/a ${ }^{18}$. Las mujeres heterosexuales alcanzan un porcentaje bastante similar $75,3 \%$ de asistencia regular. "No tengo relaciones", "no estoy enferma", "me da vagancia", "vergüenza" o sencillamente ausencia de interés fueron los argumentos más frecuentemente mencionados tal como habíamos relevado en la investigación de corte cualitativo para mujeres heterosexuales en $2007^{19}$. Estos números coinciden a su vez con lo de una investigación italiana sobre salud de lesbianas y gays que encontró que el 27,6\% de las mujeres encuestadas (de una muestra de 2.084 mujeres) no se ha realizado nunca un control ginecológico ${ }^{20}$.

Sin embargo, existe una distancia entre lo que lesbianas y bisexuales hacen, lo que los estudios cuantitativos describen y la percepción que ellas tienen en relación con las prácticas de cuidado y de atención gineco mamaria de sus pares. Casi todas las entrevistadas aducen que, en general, las lesbianas no van al ginecólogo o van menos que las heterosexuales. Mencionan parejas, amigas, conversaciones con otras mujeres lesbianas o charlas en foros de Internet como fuentes de información ${ }^{10}$. Esta discordancia entre lo que dicen los pocos datos disponibles y la autopercepción de las mujeres sobre los niveles de atención fortalece la hipótesis de que, en contextos homo/lesbofobias, en los que rigen las lógicas de la invisibilización, uno de los obstáculos más importantes para el acceso a la atención de la salud es la discriminación anticipada o sentida ${ }^{4,6,21}$.

\section{Las políticas de la invisibilización y sus lógicas}

El alejamiento de la expectativa de la heterosexualidad presupuesta en mujeres jóvenes adultas rompe con los guiones esperados en los contextos de la atención de la salud sexual y reproductiva ${ }^{5,10}$. Y se revela, por ejemplo, en el hecho de que la primera relación homosexual no está asociada a la asistencia a la consulta ginecológica como sí ocurre generalmente en el caso del inicio (su cercanía temporal) de relaciones heterosexuales ${ }^{19}$. De hecho, tres de los principales problemas para el acceso a la salud de mujeres lesbianas y bisexuales se vinculan directamente con el carácter heteronormativo que asume la consulta: a) el ocultamiento de la orientación sexo-afectiva o la gestión del secreto de la homosexualidad ${ }^{21} \mathrm{y}$, consecuentemente b) la invisibilidad de las prácticas homoeróticas femeninas así como: c) la deficiente información disponible sumada a mitos y prejuicios difundidos. 


\section{De la discriminación por orientación/práctica sexual en los servicios de salud}

La discriminación sobre la base de la orientación sexual no heterosexual es una conocida barrera al acceso al sistema de salud. Eso ya ha sido mostrado en investigaciones sobre $\mathrm{VIH} /$ sida en su relación con los gays y otros varones con prácticas homosexuales ${ }^{6,22,23}$ (confirmar se estas referências estão corretas). De las lesbianas se conoce menos ${ }^{24,25}$. En todo caso, la discriminación no es un fenómeno homogéneo y simple de describir ya que se manifiesta de diversas formas. La discriminación puede ser ejercida directa o indirectamente. Es directa cuando una norma o actitud apuntan directamente a alguna categoría de actos o personas, o cuando distinguen arbitrariamente entre categorías. La discriminación es indirecta cuando una norma o actitud son de apariencia universal, pero sus efectos discriminatorios son sufridos exclusivamente por una categoría determinada de actos o personas ${ }^{21}$. En relación con las lesbianas y mujeres bisexuales, no hay leyes o reglamentaciones que directa y explícitamente las excluyan del sistema de salud. Pero indirectamente la presunción de heterosexualidad opera en ese sentido porque invisibiliza a la vez las prácticas sexuales predominantemente llevadas a cabo por mujeres no heterosexuales así como a ellas mismas inhibiendo en el mismo movimiento la producción y circulación de información sobre ambos tópicos ${ }^{5,7}$.

Por otra parte, la discriminación puede ser real o anticipada. Es real cuando es efectivamente ejecutada, mientras que es anticipada cuando la persona, presuponiendo a un rechazo, se autodiscrimina. Cuando esto se reitera en el tiempo, se naturaliza e incluso se oculta en parte este mecanismo. ¿Por qué no se acude a consultas y controles? Porque no, es la respuesta más común.

En materia de homosexualidad, la discriminación es importante bajo su forma indirecta y como discriminación sentida o anticipada. El miedo frente a la hipotética revelación de la homosexualidad opera como una causa eficiente de auto-exclusión y de vergüenza personal. Como se trabajara ampliamente en relación con el VIH, la anticipación de la discriminación puede favorecer los riesgos e impide el acceso a los servicios de salud y sociales así como la continuidad y adherencia a los comportamientos preventivos y eventuales tratamientos ${ }^{26}$. Lo mismo ocurre de acuerdo con investigaciones realizadas en Perú y Brasil en el caso de la atención de salud a lesbianas. Se ha encontrado que una de las causas más mencionadas por las que mujeres lesbianas no concurren a los servicios de ginecología está vinculado con variables como el estigma y la discriminación ${ }^{27}$.

\section{La primera consulta. Rutinas heterosexistas}

En la primera consulta, la indagatoria de rutina se rige por la presunción heterosexista; es decir, que las mujeres tienen relaciones sexuales exclusivamente con varones y que son exclusivamente coitales vaginales. "[S]e presume que todo el mundo es heterosexual, salvo que declare lo contrario"10. Se presume, además, que la sexualidad es exclusivamente genital penetrativa. Dos preguntas clave son formuladas en el primer contacto profesional - paciente: "¿Tenés relaciones sexuales?" y en caso afirmativo se pregunta seguidamente - casi en una línea de continuidad- "¿cómo te cuidas?". El interrogante tiene un referente específico: se asocia a métodos de cuidado anticonceptivo en el marco de relaciones heterosexuales. Por ello, podría ser formulada de la siguiente manera: "¿qué método anticonceptivo usás para cuidarte?" Tal interrogatorio rutinario no se ajusta a las experiencias sexuales de las mujeres que tienen sexo con mujeres. Sus respuestas pueden incomodar si dan paso a la revelación del secreto o, cuando se opta por la negativa, puede molestar a los y las profesionales que, en clave heterosexista reproductiva entienden la respuesta negativa como un deliberado no cuidado anticonceptivo. Difícilmente haya espacio para prácticas sexuales no coitales y mucho menos, que no vinculen a otro varón en el imaginario relacional. 


\section{La revelación y la elección del/a profesional}

Para lesbianas y bisexuales, la consulta ginecológica, como cualquier otro encuentro con alguien extraño, implica confrontar el coming out. Cada nuevo encuentro es una reactualización de la pregunta acerca del modo cómo se gestiona el secreto o si puede revelarse ${ }^{21}$. Para algunas mujeres, el proceso de "blanquear" su orientación sexual frente a e/la médico/a no representa mayores problemas. Aun más, resaltan que la mayor incomodidad es la que experimenta e/la médico/a, que no espera encontrarse frente a una mujer lesbiana o bisexual en el marco de la consulta. No obstante, ir al/a ginecólogo/a y "blanquear" la orientación sexual homosexual puede implicar un esfuerzo, subjetivo y emocional, se esté visibilizada o no, ya que se inscribe en el contexto de una sociedad hegemónicamente heterosexual en el marco de la cual las prácticas sexuales y afectivas homoeróticas aun cuando puedan ser toleradas no son reconocidas ${ }^{21}$ y se hallan fuertemente invisibilizadas ${ }^{27,28}$. Por otro lado, el coming out es un proceso que se actualiza constantemente; por ende, ante cada profesional se evalúa, se piensa, y se decide hacerlo o no: depende de lo que el profesional transmita, de cuán visibilizada esté la mujer, de cómo llegó al profesional o de los vínculos de éste con otros espacios como el trabajo o la familia.

Pero además, en el caso de la consulta ginecológica, el proceso de visibilidad/ invisibilidad se encuentra condicionado por la autoridad y la desigualdad entre personal médico y pacientes, algo que resulta complicado incluso en el caso de mujeres que encarnan la norma heterosexual ${ }^{16,29}$. Esto se observa particularmente cuando el profesional es un varón ya que en esos casos las dificultades para informar la orientación homosexual son mayores según revelan nuestras entrevistadas. Y, como si se tratara de un medio para superar o disminuir la desigualdad en el vínculo, la amplia mayoría de las mujeres prefiere una ginecóloga mujer. Esos datos contrastan con lo hallado el caso de la investigación con mujeres heterosexuales exclusivamente a quienes les resultaba indistinto el sexo $\mathrm{del} / \mathrm{a}$ profesional ${ }^{15}$.

Porque se sienten más cómodas entre mujeres, porque no les gustan los varones, porque buscan una relación de "pares" o un vínculo de confianza, o porque buscan protección, cuidado y contención: son los motivos que las llevan a preferir otra mujer como profesional ginecológico. El varón genera incomodidad, rechazo, miedo, vergüenza, pudor. Aún más, la consulta ginecológica es percibida en este caso por las entrevistadas como un espacio de mujeres porque a pesar de la pretensión de abstracción con la que se consideran los cuerpos (y las personas) en las consultas médicas, se trata siempre de cuerpos sexuados, tanto de parte de las pacientes como de los y las profesionales ${ }^{19}$.

El hecho de ser mujer se privilegió sobre el hecho de compartir la orientación sexual - afectiva, probablemente porque aquello mismo que hace posible mantenerla en secreto: su no evidencia externa. Es más sencillo saber si es varón o mujer que si es o no lesbiana o bisexual, ya que, la orientación sexual puede ser manejada en función del secreto y no así la dimensión sexo genérica de las personas ${ }^{21}$. Sin embargo, elegir una ginecóloga mujer no implica revelar la orientación sexual homosexual ya que son pocas quienes afirman haber revelado su identidad homosexual como ha sido anotado en otras investigaciones mostrando las dificultades en la atención que tiene este grupo de mujeres ${ }^{27}$. Para las entrevistadas su sexo-género no es igual a sus prácticas sexuales tal como ha sido señalado en varias oportunidades ${ }^{30-32}$. Y, en consonancia con otras investigaciones ${ }^{33,34}$, las pacientes ginecológicas no suelen hablar sobre su sexualidad en el ámbito de la consulta y procuran jugar al juego propuesto que implica cierto distanciamiento erótico - afectivo entre paciente y profesional, limitándose a dialogar sobre temas que parecen competirle sólo a su cuerpo biológico (asuntos de cuidado, dolencias particulares) y sobre los que la ginecología tiene saber, como si fuera posible separar y escindir sexo, sexualidad y género ${ }^{32}$. No obstante, en el caso de las mujeres no heterosexuales 
esto funciona como un arma de doble filo porque en el marco de una sociedad hegemónicamente heterosexual que restringe la noción de sexo a la penetración coital vaginal tanto las prácticas como las identidades no hegemónicas (mucho más aún en el caso de las mujeres) quedan fuera de la visibilidad social y también biomédica. De modo que, este no decir sobre las prácticas sexuales no heterosexuales en las consultas regulares contribuye a reproducir - al tiempo que ilustra - la invisibilidad de la homosexualidad femenina: las mujeres lesbianas - o que desarrollan prácticas sexuales homoeróticas pueden ir regularmente al ginecólogo pero sus prácticas permanecen invisibles.

\section{La invisibilidad del sexo entre mujeres}

Permeado por los estereotipos falocéntricos que vinculan el sexo con la penetración del pene, subyace la idea que las lesbianas no tienen sexo. Por ende, no se requieren cuidados sexuales ya que en las culturas heterosexistas reproductivas éstos sólo son pensados en términos coitales. La asociación entre el sexo entre mujeres y las prácticas de cuidado va por la negativa: si se tienen relaciones sexuales con mujeres no es necesario cuidarse. Sin embargo, el no cuidado y el tener sexo con mujeres no son lo mismo: las mujeres que tienen sexo con mujeres pueden implementar - y es aconsejable que lo hagan - prácticas de cuidado (Meinerz, 2005). Son prácticas de cuidado entre mujeres lavarse las manos, protegerlas de heridas, tomar recaudos para la penetración manual o con objetos o, bien para practicar el sexo oral. Asimismo, aún cuando en otro orden, se encuentran los controles ginecológicos ${ }^{27}$.

La idea acerca de la innecesariedad de cuidados en las prácticas sexuales entre mujeres no es privativa de la cultura heterosexual. Lesbianas y bisexuales suelen compartir este prejuicio que funciona como una importante barrera para el acceso a la salud para esta población así como también ocurre con los casos de mujeres no iniciadas sexualmente, o las mujeres con voto de castidad.

\section{La invisibilidad lésbica a través de la ausencia de saber}

Otro de los obstáculos documentados - y que se suceden sólo luego que la mujer salga del clóset frente al profesional y por ende sólo en tales casos - es que la información sobre la no heterosexualidad de la paciente no ocasiona ningún tipo de intervención, consejo, información o cuidados particulares porque, como señala ${ }^{8}$, las lesbianas han sido invisibilizadas en la mayoría de los campos de saber hasta no hace mucho. Decirlo puede o no generar cierta incomodidad inicial pero esto no afectaría en lo sustancial el vínculo asistencial y terapéutico ya que aún en los casos de profesionales abiertos, prima la ausencia de información clara y pertinente para darle a las personas cuyas prácticas sexuales se efectúan fuera del patrón erótico hegemónico. Quizás el silencio sea uno de los principales problemas en el vínculo asistencial. No la discriminación explícita, el rechazo a la atención o los malos tratos, sino el silencio. No tener nada para decir por no haber sido capacitado/a en el tema, por no saber cómo reaccionar, por no saber cómo resolver o qué decir. Es el silencio del desconocimiento, de no tener información para ofrecer o espacio para alojar ${ }^{28,34}$.

Frente al silencio y la invisibilización que supone y reproduce, otras, unas pocas mujeres, reivindican algo semejante a una actitud militante: es preciso decir que se tienen prácticas homosexuales, es preciso que la lesbiana se muestra en escena, se visibilice. La visibilización en este caso no vincula tanto con la intención de recibir una mejor o más adecuada atención o por la calidad del acto asistencial sino como un modo para hacer presente el lesbianismo en el marco de la consulta y provocar que el médico "aprenda", amplíe su conocimiento, o hacer un "cortocircuito en esa normalidad". 


\section{Conclusiones abiertas}

La heteronormatividad y su presunción, la desigualdad de género y la asimetría médico/a-paciente podrían actuar como barreras a la revelación de la homosexualidad en el ámbito de la consulta. Del mismo modo, la invisibilidad de las prácticas homoeróticas femeninas podría contribuir a mantener este carácter secreto, íntimo y silenciado. Sin embargo, los datos analizados también muestran que para las entrevistadas se presenta como innecesario comunicar su orientación sexual: "No hay necesidad de decirlo" "no me parecía que me pudiera resolver o aportar nada" "no tiene nada que ver", "no me parece que aporte nada ni a él ni a mí".

Se da una especie de división de la mujer entre su cuerpo biológico y su sexualidad lo que daría una invisibilidad de su identidad sexual; es decir, se peformaría su ser mujer como independiente de su ser sexual - heterosexual, lesbiana o bisexual. Así, no sería necesario revelar las prácticas sexuales porque éstas no modifican ni la morfología, ni la fisiología ni la biología corporal que es el objeto de la atención médica ginecológica. El cuerpo biológico de la mujer estaría separado de sus prácticas sexuales, eróticas afectivas - todas ellas sociales. Pero no es mera biología lo que se atiende en una consulta ginecológica, aunque se cuiden, curen o controlen los cuerpos de las mujeres. El cuerpo de la mujer que se presenta y se atiende en las consultas ginecológicas es un cuerpo heterosexual. Con necesidades de cuidado y salud de prácticas sexuales heterosexuales, con información, resguardos y asesoramiento de prácticas heterosexuales - aun cuando no se hable de sexualidad en la consulta.

De lo anterior se desprende que es necesario visibilizar la no heterosexualidad femenina en el campo social en general y en particular en el médico porque no hay cuerpo sin sexo-género ni sexo-género sin soporte material - cuerpo, en este caso. Aún más, es necesario poner en circulación las múltiples prácticas sexuales que pueden tener lugar entre dos personas independientemente de su sexo y orientación sexual y sus correspondientes cuidados a la hora de practicarlos, ya que el sexo oral o la penetración con objetos, por ejemplo, no son privativas de las personas no heterosexuales. Finalmente, probablemente, sea mucho más productivo enfocar las políticas hacia el conjunto de prácticas sexuales posibles independientemente de la identidad sexual de las personas para evitar exclusiones o discriminaciones innecesarias.

\section{Colaboradores}

Las y los autores trabajaron juntos en todas las partes del manuscrito. 


\section{Referencias}

1. Brown J. Mujeres y ciudadanía en Argentina: debates teóricos y políticos sobre derechos (no) reproductivos y sexuales (1990-2006). Buenos Aires: Teseo; 2013.

2. Pecheny M, Tamburrino C, Mario S, Luciano Conde L, Capriati A, Brown J, et al. Informe Salud Investiga: salud sexual y reproductiva: problemas emergentes. Buenos Aires: Salud Investiga, Ministerio de Salud de la Nación; 2011.

3. Epele M. Etnografía, fragmentación social y drogas: hacia una política de las miradas. Etnogr Contemp. 2007; 3(3):117-43.

4. Careaga G, Pessah M. La experiencia lésbica, la sexualidad y la salud [Entrevista de Ciudadanía Sexual] [acceso 2013 Dic 8]. Bol Ciud Sexual. 2005; 16(2). Disponible en: http://www.ciudadaniasexual.org/boletin/b16/articulos.htm\#3

5. Vergara Sánchez PK. El viaje de las invisibles. Manifestaciones del Régimen Heterosexual en experiencias de mujeres lesbianas en consultas ginecológicas [tesis]. México (DF): Universidad Autónoma Metropolitana; 2013.

6. Ortíz Hernández L. Revisión crítica de los estudios que han analizado los problemas de salud de bisexuales, lesbianas y homosexuales. Salud Problema. 2004; 16(9):19-39.

7. Figari C, Gemetro F. Escritas en el silencio: mujeres que deseaban a otras mujeres en la Argentina del siglo XX. Sexual Salud Soc. 2009; 3:33-53.

8. Gemetro F. Figuraciones lésbicas en las primeras producciones de las ciencias sociales. In: 8a Reunión de Antropología del Mercosur - Diversidad y poder en América Latina; 2009; Buenos Aires, Argentina. Buenos Aires: RAM; 2009.

9. Fuskova I, Schmid S, Marek C. Amor de mujeres. El lesbianismo en la Argentina, hoy. Buenos Aires: Planeta; 1994.

10. Espartiles. Cambiemos las preguntas: campaña nacional por una atención digna de las lesbianas y mujeres bisexuales en los sistemas de salud [Internet] 2006 [acceso 2013 Jul 30]. Disponible en: http://www.clam.org.br/pdf/cambiemos.pdf

11. Ariza L, Schwartz P. Relaciones amorosas y parentalidad en población GLTTBI. In: Jones D, Libson M, Hiller R, editores. Sexualidades, política y violencia: Marcha del Orgullo GLTTBI Buenos Aires 2005. Buenos Aires: Antropofagia; 2006. p. 51-63.

12. Rodríguez Gómez G, Gil Flores J, García Jimenez E. Metodología de la investigación cualitativa. Málaga: Ediciones Aljibe; 1996.

13. Huberman A, Miles M. Data management and analysis methods. In: Denzin N, Lincoln Y, editors. Handbook of qualitative research. London: Sage; 1994. p. 769-802.

14. Luciani Conde L, Barcala A. Niñez en condiciones de desamparo y acceso a la salud en Ciudad de Buenos Aires. In: 15o Anuario de Investigaciones de la Facultad de Psicología; 2007; Buenos Aires. Buenos Aires; 2007. p. 89-116.

15. Brown J, Tamburrino C, Pecheny M. Salud Sexual y reproductiva: la noción de autonomía de las mujeres puesta en cuestión. El aborto y otras situaciones sensibles. In: Actas de las IV Jornadas de Investigación en Antropología Social; 2006; Buenos Aires, Argentina. Buenos Aires: Instituto de Antropología Social-FFyL - UBA; 2006.

16. Carvalho ML, Furegato, AR. Exame ginecológico na perspectiva das usuárias de um serviço de saúde [Internet]. Rev Eletr Enfedrm. 2001; 3(1) [acceso 2013 Ago 8]. Disponible en: http://www.revistas.ufg.br/index.php/fen/article/view/698 
17. Ehrenreich B, English D. Brujas, comadronas y enfermeras: historia de las sanadoras dolencia y trastornos: política sexual de la enfermedad. Barcelona: Ediciones La Sal; 1988.

18. Brown J. Salud ginecológica. In: Jones D, Libson M, Hiller R, editores. Sexualidades, política y violencia: Marcha del Orgullo GLTTBI, Buenos Aires; 2005. Buenos Aires: Antropofagia; 2006. p. 93-102.

19. Brown J, Gattoni S, Tamburrino C, Pecheny M. La primera experiencia es la que cuenta: notas sobre la trayectoria de pacientes ginecológicas. In: Actas del 260 Congreso de la Asociación Latinoamericana de Sociología, Guadalajara; México; 2007. Guadalajara: ALAS; 2007. p. 1-17.

20. Arcigay. Survey nazionale su stato di salute, comportamenti protettivi e percezione del rischio HIV nella popolazione omo-bisessuale. Bologna: Associazione Lesbica e Gay Italiana; 2006.

21. Pecheny M. Identidades discretas. In: Arfuch $L$, compilador. Identidades, sujetos $y$ subjetividades: narrativas de la diferencia. Buenos Aires: Prometeo; 2002. p. 5-17.

22. Parker R, Aggleton P. HIV and Aids-related stigma and discrimination: a conceptual framework and implications for action. Rio de Janeiro: ABIA; 2002.

23. Manzelli $H$, Pecheny M. HIV/Aids prevention in men who have sex with men. In: Cáceres $C$, Pecheny $M$, Terto Junior $V$, editors. Aids and male-to-male sex in Latin America: vulnerabilities, strengths and proposed measures - perspectives and reflections from the point of view of public health, social sciences and activism. Lima: UPCH/UNAIDS; 2003. p. 156-82.

24. Guimaraes CD. "Mas eu conheço ele!" Um método de prevenção do HIV/ Aids. In: Parker R, Galvao J, organizadores. Quebrando o silêncio: mulheres e Aids no Brasil. Rio de Janeiro: Relume Dumará; 1996. p. 169-79.

25. Arendt ED. The politics of invisibility: HIV-positive women who have sex with women and their struggle for support. Int J Assoc Nurses AIDS Care. 2003; 14(6):37-47.

26. Pecheny $M$, Manzelli $H$. Estudio nacional sobre la Situación social de las personas viviendo con VIH en la Argentina. Buenos Aires: Libros del Zorzal; 2008.

27. Galarreta ME. [Internet] La salud de las lesbianas: apuntes desde la experiencia de atención. In: Boletín Ciudadanía Sexual. Lima: Ciudadanía sexual; 2005 [acceso 2014 Jun 14]. Disponible en: http://www.ciudadaniasexual.org/boletin/b16/articulos.htm\#3

28. Faccini R. [Internet] Mujeres, homosexualidades y salud: visibilizando demandas e caminos. In: Boletín Ciudadanía Sexual. Lima: Ciudadanía sexual; 2005 [acceso 2014 Jun 14]. Disponible en: http://www.ciudadaniasexual.org/boletin/b16/articulos.htm\#3

29. Pecheny M, Alonso JP, Brown JL, Capriati A, Mantilla J, Gattoni S, et al. La ciudadanización de la salud: derechos y responsabilidades en salud sexual-reproductiva, enfermedades crónicas y cuidados paliativos. In: López E, Pantelides E, compiladoras. Aportes a la investigación social en salud sexual y reproductiva. Buenos Aires: CENEPCEDES-AEPA-UNFPA; 2006. p. 64-79.

30. Viñuales $\mathrm{O}$. Cadena simbólica: relación entre sexo, género, prácticas sexuales y orientación sexual [Internet]; 2008 [acceso 2011 Mayo 7] Disponible en: http://api.ning.com/files/IC84NzWyG7m0aBNzPkGWrtkVZ4gINIVva24SiCbM W4*TO9LxpXKihGxcBqnkXH2w*T4qL-pkOyMYVunxgOBWeSHyWPG1keR*/ OlgaViualesCADENASIMBLICAUimp2008.pdf 
31. Viñuales O. [Internet] Poder y deseo: relaciones de dominación y sumisión. 2008 [acceso 2011 Mayo 7]. Disponible en: http://www.amicsgais.org/html/ articles/poder_y_deseo.html

32. Butler J. Cuerpos que importan: sobre los límites materiales del sexo. Buenos Aires: Paidós; 2006.

33. Galansinski D, Ziólkowska J. Gender and the gynecological examination: women's identities in doctors' narratives. Qual Health Res. 2007; 17(4):477-88.

34. Gattoni S, Brown J, Tamburrino C, Pecheny, M. ¿La primera experiencia es la que cuenta? Notas sobre la trayectoria de pacientes ginecológicas. In: Actas de VII Jornadas Nacionales de Debate Interdisciplinario en Salud y Población; 2007; Buenos Aires, Argentina. Buenos Aires: Instituto de Investigaciones Gino Germani, Facultad de Ciencias Sociales, UBA; 2007. p. 18.

35. Barbosa R, Faccini R. Saúde das mulheres lésbicas: promoção da equidade e da integralidade. São Paulo: Fundación Ford, UNFPA; 2006.

Brown JL, Pecheny M, Tamburrino MC, Conde LL, Perrotta GV, Capriati A, et al. Cuidados ginecológicos em mulheres lésbicas e bissexuais: notas sobre a situação na Argentina. Interface (Botucatu). 2014; 18(51):673-84.

$\mathrm{O}$ artigo apresenta resultados de uma pesquisa qualitativa sobre questões emergentes em saúde sexual e reprodutiva, cujo objetivo foi descrever as experiências de mulheres não heterossexuais em relação aos serviços de saúde sexual e reprodutiva. O objetivo do trabalho foi identificar e analisar as percepções de obstáculos e facilitadores do acesso aos serviços de saúde. Foram realizadas 18 entrevistas semidirigidas com mulheres não heterossexuais e os resultados principais incluíram: o obstáculo específico ao acesso à saúde sexual e reprodutiva das mulheres não heterossexuais deriva de uma "lógica da invisibilidade" dessa população como paciente ginecológica, que resulta na antecipação da discriminação (discriminação sentida) num contexto homo / lesbofóbico, na ausência de produção teórica e intervenções práticas sobre saúde e sexo entre mulheres, e na invisibilidade das lésbicas e bissexuais como coletivo.

Palavras-chave: Sexualidade. Reprodução. Serviço de saúde reprodutiva. Bissexualidade. Homossexualidade feminina.

Recebido em 22/04/13. Aprovado em 09/07/14. 